\title{
A SYNTHETIC CHARACTERIZATION OF THE HEMISPHERE
}

\author{
CHRISTOPHER B. CROKE
}

(Communicated by Jon G. Wolfson)

\begin{abstract}
We show that round hemispheres are the only compact two-dimensional Riemannian manifolds (with or without boundary) such that almost every pair of complete geodesics intersect once and only once. We prove this by establishing a sharp isoperimetric inequality for surfaces with boundary such that every pair of geodesics has at most one interior intersection point.
\end{abstract}

\section{INTRODUCTION}

In this short note we prove a sharp isoperimetric inequality for two-dimensional Riemannian manifolds $(M, \partial M, g)$ with boundary $\partial M$. The result will apply to surfaces such that every pair of complete geodesics has at most one interior intersection point. So in particular it applies to surfaces where all geodesics are minimizing. By a "complete geodesic" we mean a curve satisfying the geodesic equation which either runs from a boundary point to a boundary point or is infinitely long and the boundary points of the curve are precisely the intersection points of the curve with the boundary. They can a priori have 0,1 , or 2 boundary points. However the intersection condition (along with the finiteness of the area) will quickly allow us to conclude that all geodesics are compact segments between two boundary points. We should point out that we are ruling out closed geodesics since they are interpreted to intersect any geodesic infinitely often if they intersect at all. (Note that we would have to include round projective planes if one interpreted such an intersection as a single intersection.)

Theorem 1.1. Let $(M, \partial M, g)$ be a two-dimensional, finite area, Riemannian manifold with (possibly empty) boundary, $\partial M$, such that every pair of complete geodesics has at most one interior point of intersection. Then

$$
L(\partial M)^{2} \geq 2 \pi \operatorname{Area}(M) .
$$

Further equality holds if and only if every pair of geodesics intersects.

This is a new proof of (and an extension of) the inequality in the following case of the isoperimetric inequality Theorem 11 of $[\mathrm{Cr}]$ :

Theorem 1.2. Let $(M, \partial M, g)$ be a two-dimensional Riemannian manifold such that all geodesics minimize until they hit the boundary. Then

$$
L(\partial M)^{2} \geq 2 \pi \operatorname{Area}(M) .
$$

Received by the editors January 23, 2007.

2000 Mathematics Subject Classification. Primary 53C22.

Supported by NSF grants DMS 02-02536 and 07-04145.

(c) 2007 American Mathematical Society 
Further equality holds if and only if $M$ is isometric to a round hemisphere.

In fact Theorem 11 of $[\mathrm{Cr}]$ says much more than this, and applies in higher dimensions.

The interest in Theorem 1.1 is twofold. First, the equality cases in the above give us the characterization of hemispheres:

Corollary 1.3. Round hemispheres are the only finite area, two-dimensional, Riemannian manifolds (with or without boundary) such that almost every pair of complete geodesics intersects once and only once.

In the infinite volume case both Theorem 1.1 and Corollary 1.3 are false because we would have to include the flat plane. In fact, below is a (probably quite difficult) question that has been open for some time.

Question 1.4. Is the Euclidean metric the only complete metric on the plane with no conjugate points such that for every line l (complete geodesic) and point $p$ not on $l$ there is a unique line through p parallel to (i.e. not intersecting) $l$ ?

The answer would be yes if there was a positive answer to:

Question 1.5. Is the Euclidean metric the only complete metric on the plane such that almost every pair of geodesics intersects at exactly one point?

Some results on Question 1.4 can be found in [Bu-Kn]. Question 1.5 was also posed by Knieper in an MSRI problem session in the early nineteen-nineties.

We should point out that although there can be no direct higher-dimensional analogue of Corollary 1.3 the closest (by using Crofton's formula below) would be: n-dimensional hemispheres are the only compact manifolds with boundary such that all complete geodesics have the same length. This was proved in [Ba] using the Blaschke conjecture for spheres. It also follows from Santaló's formula (see below) and the equality case of Theorem 11 of $[\mathrm{Cr}]$. The above characterization is indeed very much related to the Blaschke conjecture. Theorem 11 of $[\mathrm{Cr}]$ is proved using the Berger-Kazdan inequality that was used in the proof of the Blaschke conjecture for spheres (see appendices of $\mathrm{D}$ and $\mathrm{E}$ of $[\mathrm{Be}]$ ). (The two-dimensional case was proved by L. Green - also see [Be].) On the other hand (as was pointed out to the author by Z. I. Szabó) the above characterization can be viewed as a stronger version of the two-dimensional case of the Blaschke conjecture, since a closed geodesic on a two-dimensional Blaschke sphere will divide the space into two parts each of which satisfies the condition that almost every pair of complete geodesics intersect once and only once.

The second reason for interest in Theorem 1.1 is that the proof tells us that the isoperimetric deficit $L(\partial M)^{2}-2 \pi$ Area $(M)$ is $\frac{1}{4}$ the measure of the set of pairs of geodesics that do not intersect (i.e. $\frac{1}{4} \operatorname{Vol}(\Gamma \times \Gamma-I)$ ). This will follow from Proposition 2.1 of the next section. In particular, if $M$ in addition has nonpositive curvature, then (see [We]) it satisfies the classical isoperimetric inequality, $L(\partial M)^{2} \geq 4 \pi \operatorname{Area}(M)$, and hence for such metrics $\frac{1}{4} \operatorname{Vol}(\Gamma \times \Gamma-I) \geq 2 \pi \operatorname{Area}(M)$ with equality holding only for the flat disc.

\section{Proofs}

We will assume throughout this section that $M$ is a surface of finite area with (possibly empty) boundary $\partial M$. We can assume that the boundary is compact for 
if not it would have infinite length and the inequality would be clearly true (and not sharp). The unit tangent bundle, $U M$, of $M$ has a natural measure, $d u$, that is locally a product measure. In particular $\operatorname{Vol}(U M)=2 \pi \operatorname{Area}(M)$. Let $\tau$ be a curve in $M$ with arclength $s$ and unit normal $\nu$. We can let $\{(\theta, s)\}$ parameterize the set of geodesics $\gamma$ that intersect $\tau$ by choosing $(\theta, s)$ such that $\gamma(0)=\tau(s)$ and the unit tangent $\gamma^{\prime}(0)$ makes angle $\theta$ with $\nu$. For all $t$ such that $\gamma(t)$ is defined we let $\{(\theta, s, t)\}$ correspond to the unit vector $u=\gamma^{\prime}(t)$. Then Santaló's formula tells us that $d u=|\cos (\theta)| d \theta d s d t$. We will be using this in a number of ways.

First consider metrics where any two geodesics intersect at most once in the interior. Note that this will follow (by continuity) if we assume that almost every pair intersects at most once. Some pairs may intersect more than once but then only at boundary points (as is the case for round hemispheres). We claim that all complete geodesics are segments between boundary points. If not, then let our infinite length geodesic play the role of $\tau$ above. We first note that $\tau:[0, \infty) \rightarrow M$ does not have a limit point on the boundary since if so a geodesic leaving normal to the boundary at that point would intersect $\tau$ more than once. In particular there is an $\epsilon>0$ such that $d(\tau[1, \infty), \partial M)>\epsilon$. Since every geodesic $\gamma$ that intersects $\tau$ intersects it only once, the parametrization $(\theta, s, t)$ for $1 \leq s<\infty$ and $-\epsilon<t<\epsilon$ of $U M$ is one-to-one (though not onto). Hence $\operatorname{Vol}(U M) \geq 4 \times \infty \times 2 \epsilon$, a contradiction.

We now consider the case where all geodesics go between boundary points (without assuming geodesics intersect at most once). It is easy to describe the (standard) measure space, $\Gamma$, of complete unit speed oriented geodesics by using the boundary $\partial M$ as our $\tau$ above. The measure is $d \gamma=|\cos (\theta)| d \theta d s$. Note that the parametrization $(\theta, s, t)$ of $U M$ is one-to-one and onto if $-\frac{\pi}{2} \leq \theta \leq \frac{\pi}{2}, s$ is an arc length parametrization of the boundary (which is not assumed to be connected) and $0 \leq t \leq L(\gamma)$ (where $\gamma$ is the geodesic determined by $(\theta, s)$ ). In particular, $2 \pi \operatorname{Area}(M)=\operatorname{Vol}(U M)=\int_{\Gamma} L(\gamma) d \gamma$.

Now consider a curve $\tau$. We claim that $\int_{\Gamma} i(\tau, \gamma) d(\gamma)=4 L(\tau)$ where $i(\tau, \gamma)$ represents the number of times the geodesic $\gamma$ intersects $\tau$. (This is known as Crofton's formula.) It follows directly from Santaló's formula when we note that the parametrization $(\theta, s)$ of the unit vectors along $\tau$ counts each $\gamma \in \Gamma$ exactly as often as $i(\tau, \gamma)$ and hence $4 L(\tau)=\iint|\cos (\theta)| d \theta d s=\int_{\Gamma} i(\tau, \gamma) d \gamma$. In particular (since each geodesic hits $\partial M$ twice) we see that $\operatorname{Vol}(\Gamma)=2 L(\partial M)$.

Proposition 2.1. Let $(M, \partial M, g)$ be such that every complete geodesic hits the boundary at both ends. Then

$$
8 \pi \operatorname{Area}(M)=\int_{\Gamma \times \Gamma} i\left(\gamma_{1}, \gamma_{2}\right) d \gamma_{1} d \gamma_{2} .
$$

In particular, if every pair of geodesics intersects at most one interior point, then $8 \pi \operatorname{Area}(M)=\operatorname{Vol}(I)$ where $I \subset \Gamma \times \Gamma$ represents the subset of geodesic pairs that intersect.

Proof.

$$
\begin{gathered}
\int_{\Gamma \times \Gamma} i\left(\gamma_{1}, \gamma_{2}\right) d \gamma_{1} d \gamma_{2}=\int_{\Gamma}\left\{\int_{\Gamma} i\left(\gamma_{1}, \gamma_{2}\right) d \gamma_{1}\right\} d \gamma_{2} \\
=\int_{\Gamma} 4 L\left(\gamma_{2}\right) d \gamma_{2}=8 \pi \operatorname{Area}(M) .
\end{gathered}
$$


Note that Theorem 1.1 follows immediately from this since $\operatorname{Vol}(I) \leq$ $\operatorname{Vol}(\Gamma \times \Gamma)=4 L(\partial M)^{2}$ and equality holds if and only if $\Gamma \times \Gamma-I$ has measure 0 . By continuity this implies that every pair of geodesics must intersect.

We now prove Corollary 1.3. First note that there can be no pair of conjugate points along any geodesic segment except for the endpoints; for if so it is easy to see that there are nearby geodesics that intersect twice in the interior and hence a set of positive measure of geodesic pairs that intersect more than once. We also note that the exponential map from any interior point is one-to-one. Although (at least a priori) this may not mean all geodesics minimize, this is exactly what is used in the proof of Theorem 11 of $[\mathrm{Cr}]$. Thus we can apply the equality case in that theorem to get the corollary.

\section{REFERENCES}

[Ba] V. Bangert, Manifolds with geodesic chords of constant length, Math. Ann. 265 (1983), no. 3, 273-281. MR721397 (85d:53016)

[Be] A. Besse, Manifolds all of Whose Geodesics are Closed, Ergebisse Grenzgeb. Math., no. 93, Springer, Berlin, 1978. MR496885 (80c:53044)

[Bu-Kn] K. Burns and G. Knieper, Rigidity of surfaces with no conjugate points, J. Diff. Geom. 34 (1991), no. 3, 623-650. MR1139642 (92j:53015)

[Cr] C. Croke, Some isoperimetric inequalities and eigenvalue estimates, Ann. Scient. Ec. Norm. Sup., 4e serie, t.13(1980), 419-435. MR608287 (83d:58068)

[C-D] C. Croke and N. Dairbekov, Lengths and volumes in Riemannian manifolds, Duke Mathematical Journal 125 (2004), no. 1, 1-14. MR2097355 (2005k:53045)

[We] A. Weil, Sur les surfaces à courbure negative, C.R.A.S. 182 (1926), 1069-1071.

Department of Mathematics, University of Pennsylvania, Philadelphia, Pennsylvania 19104-6395

E-mail address: ccroke@math.upenn.edu 